\title{
Assessment of advanced glycated end product accumulation in skin using auto fluorescence multispectral imaging
}

Marcus Larsson, Riccardo Favilla and Tomas Strömberg

\author{
Linköping University Post Print
}

\section{Tweet}

N.B.: When citing this work, cite the original article.

Original Publication:

Marcus Larsson, Riccardo Favilla and Tomas Strömberg, Assessment of advanced glycated end product accumulation in skin using auto fluorescence multispectral imaging, 2015, Computers in Biology and Medicine.

http://dx.doi.org/10.1016/j.compbiomed.2016.04.005

Copyright: Elsevier

http://www.elsevier.com/

Postprint available at: Linköping University Electronic Press

http://urn.kb.se/resolve?urn=urn:nbn:se:liu:diva-128653

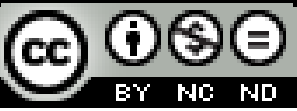




\section{Assessment of advanced glycated end product \\ accumulation in skin using auto fluorescence multispectral imaging}

\section{Authors and Affiliations}

Marcus Larsson (corresponding author)

Department of Biomedical Engineering

Faculty of Science and Engineering

Linköping University

Sweden

\section{Riccardo Favilla}

Institute of Clinical Physiology

Council of National Research

Pisa

Italy

\section{Tomas Strömberg}

Department of Biomedical Engineering

Faculty of Science and Engineering

Linköping University

Sweden

Corresponding author contact information

Name: Marcus Larsson

Address: Department of Biomedical Engineering

Linköping University

58183 Linköping

Sweden

Email: marcus.larsson@liu.se

Phone: $\quad$ +4613286751

Fax: $\quad$ +4613101902 


\section{Keywords}

Advanced glycation end products; multi spectral imaging; noninvasive; auto fluorescence; selfmonitoring

\section{Abstract (250 words)}

Several studies have shown that advanced glycation end products (AGE) play a role in both the microvascular and macrovascular complications of diabetes and are closely linked to inflammation and atherosclerosis. AGEs accumulate in skin and can be detected using their auto fluorescence (AF).

A significant correlation exists between AGE AF and the levels of AGEs as obtained from skin biopsies. A commercial device, the AGE Reader, has become available to assess skin AF for clinical purposes but, while displaying promising results, it is limited to single-point measurements performed in contact to skin tissue. Furthermore, in vivo imaging of AGE accumulation is virtually unexplored.

We proposed a non-invasive, contact-less novel technique for quantifying fluorescent AGE deposits in skin tissue using a multi-spectral imaging camera setup (MSI) during ultraviolet (UV) exposure. Imaging involved applying a region-of-interest mask, avoiding specular reflections and a simple calibration. Results of a study conducted on 16 subjects with skin types ranging from fair to deeply pigmented skin, showed that AGE measured with MSI in forearm skin was significantly correlated with the AGE reference method (AGE Reader on forearm skin, $R=0.68, p=0.005)$. AGE measured in facial skin was borderline significantly related to AGE Reader on forearm skin ( $R=0.47, p=0.078)$. These results support the use of the technique in devices for non-touch measurement of AGE content in either facial or forearm skin tissue over time. 


\section{Introduction}

Advanced glycation end-products (AGEs) are long-term indicators of metabolic and glycemic stress that can be found in human skin. They derive from the modifications of proteins or lipids that after contact with aldose sugars become glycated. [1]. Factors that affects the formation of AGEs are: extended periods of hyperglycemia; oxidant stress in the cellular environment; and the rate of turnover of proteins for glycoxidation.[2]

AGEs can also be absorbed through the diet. [3] Foods high in protein and fat are especially rich in AGEs. In addition, increased cooking temperatures, like broiling and frying, and increased cooking times lead to increased amounts of AGEs. [4]

Inside the tissue, AGEs can alter cell structure and function, contribute to diabetes related micro- and macrovascular complications [5], and may modify the extracellular matrix. [6] They may also lead to the release of free radicals [6], block the activity of nitric oxide inside the endothelium [7] and increase the amount of reactive oxygen species. [8] When the AGEs have been formed inside the tissue, their turnover time is very long.

Considering the negative effects of AGEs in tissue, an objective way of quantify such an accumulation is of clinical value. Since several AGEs exhibit characteristic fluorescence, this physical effect can be exploited to perform a non-invasive assessment of accumulation increases into human tissues. Significant correlations have been found between skin autofluorescence (AF) and levels of skin AGEs like pentosidine, as obtained from skin biopsies when studying e.g. diabetes mellitus.[9] Skin AF can be used as a predictor for assessing how diseases with increased cardiovascular risk develops. Koetsier et al [10] suggest that UV stimulations by a broad excitation range of $355-405 \mathrm{~nm}$, is adequate for inducing AF for diagnostic purposes. 
A commercial device, the AGE Reader (Diagnoptics Technologies B.V., Groningen, The Netherlands), has become available to assess skin auto fluorescence for clinical purposes.[11] A black light tube, with a peak wavelength of $370 \mathrm{~nm}$ is used to illuminate a small region of the skin on the forearm. An optical fiber detects the emission and reflected excitation light, and a spectrometer is used to measure the intensity spectrum. This device is limited to single-point measurements performed in contact to forearm skin.

The aim of this work is to demonstrate the feasibility of quantifying fluorescent AGE deposits in skin tissue using a two-camera setup with optical band pass filters during ultra-violet (UV) exposure. Such a tool enables the analysis of the spatial distribution of AGE in tissue and can be used in devices devoted to remote monitoring and self-monitoring whenever trends in AGE tissue content are required to be traced over time, such as a smart mirror. Incorporating physiological measurements in a smart mirror will increase its medical value [12]. As a proofof-concept, the AGE level quantified with the proposed camera setup will be compared to readings from a commercial instrument for single point measurements performed in contact to arm skin tissue.

\section{Material and Methods}

\section{AGE auto fluorescence and quantification}

The possibility for detecting the AGE related auto fluorescence spectrum in an imaging setup was initially evaluated in a pilot study using a HSI (Hyper Spectral Imaging) system consisting of a monochromatic camera (Dolphin F-145B, Allied Vision Technologies GmbH, Germany) with an attached tuneable LCTF (Liquid Chrystal Tuneable Filter; VariSpec VIS 7nm FWHM, PerkinEImer Inc., US) band-pass filter. This setup was capable of capturing HSI data in the 445- 
720nm range. The initial evaluation encompassed HSI data taken from the-volar side of the forearm during UV exposure (365nm; R130-365-N, Smart Vision Lights, US) using LED light below $400 \mathrm{~nm}$ (Figure 1). In total, 18 subjects were evaluated. This data display a consistent spectral appearance similar to that of AGE auto fluorescence $[13,14]$, with a peak located at 480-490 nm (Figure 2). This result indicates that an MSI camera setup capable of capturing the light above 450nm would be suitable for detecting UV induced AGE auto fluorescence. In order to minimize the influence from the strong blood-related absorption peaks above $500 \mathrm{~nm}$, the detection of auto fluorescence should be limited to the 450-500nm spectral band.

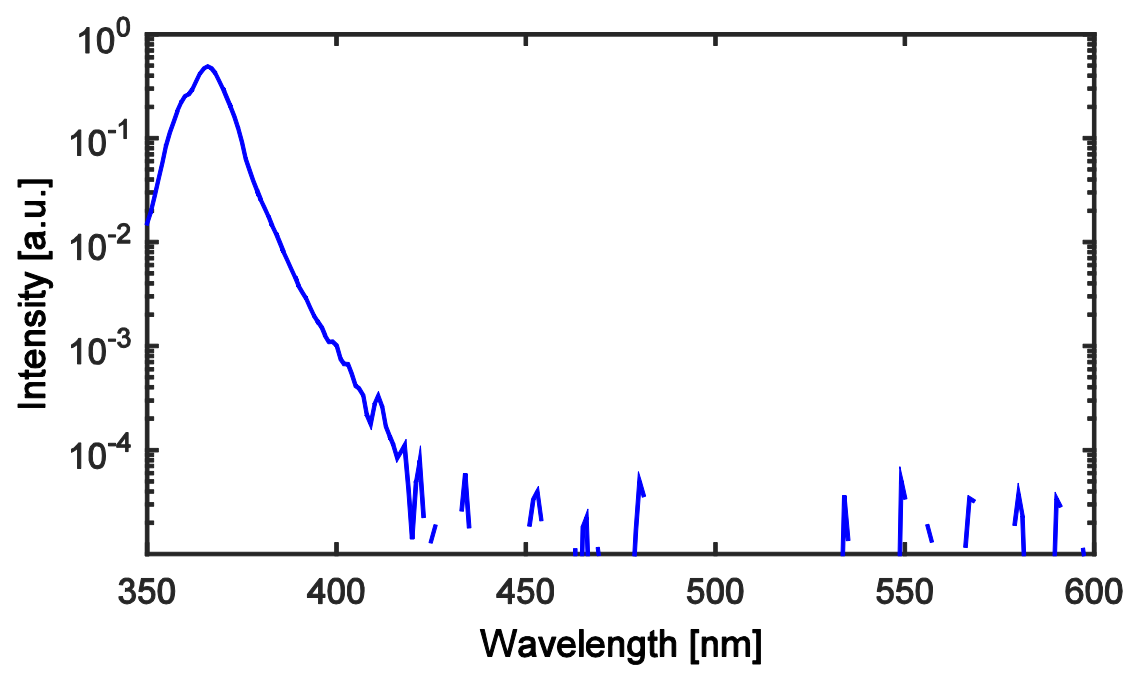

Figure 1. Emission spectra of the $365 \mathrm{~nm}$ UV LED excitation light source. 


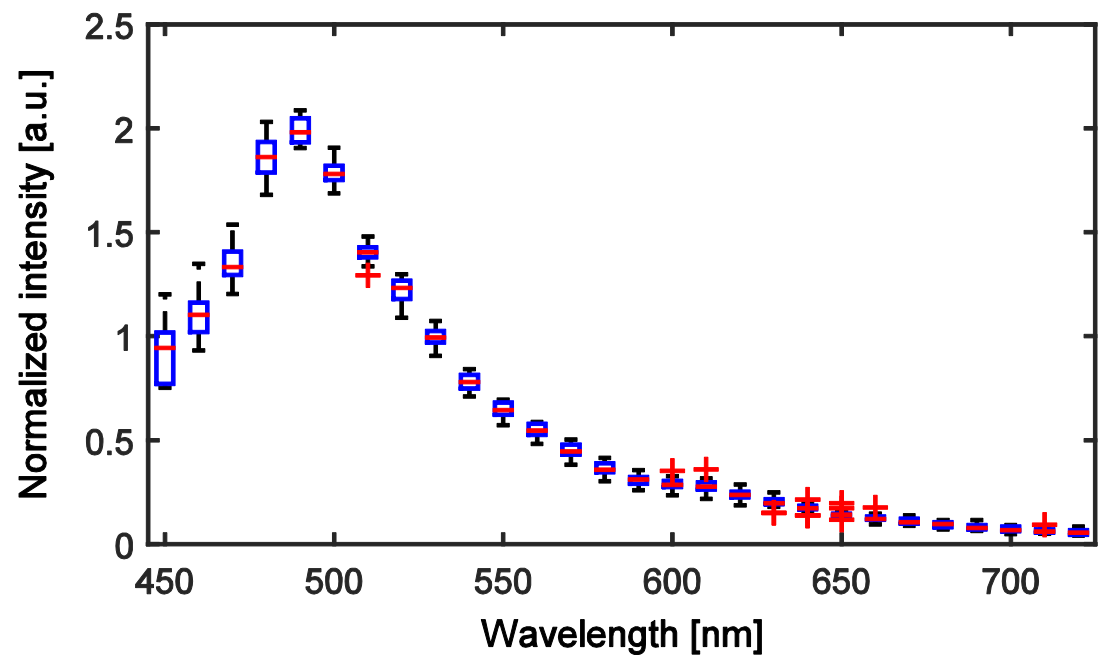

Figure 2. Box-plot of 18 auto fluorescence spectra, each normalized by their average intensity. The spectra were captured from the volar side of the forearm when exposed to 365nm UV LED light. An auto fluorescence peak is shown at $480-490 \mathrm{~nm}$.

The amount of backscattered auto fluorescence depends not only on the amount of fluorophores in tissue but also on the intensity of the excitation UV light. A simple, yet effective way of quantifying the amount of excitation light is to detect the diffusely backscattered UV light. As proposed by Graaff et al. [13], the excitation intensity dependency can be canceled out by calculating the normalized auto-fluorescence ratio

$$
A F=\frac{I_{e m}}{I_{e x}},
$$

where $I_{e m}$ is the mean detected auto fluorescence $(450-500 \mathrm{~nm})$ and $I_{e x}$ is the mean detected diffusely backscattered UV light. Using a $365 \mathrm{~nm}$ UV LED light source, $I_{e x}$ can be quantified by detecting light below 400nm. 
The multi-spectral imaging camera setup (Figure 3) consisted of two monochromatic cameras (FL3-U3-32S2M-CS, Point Grey Research Inc., CA) placed closely beside each other, about $35 \mathrm{~mm}$ apart (center-center separation), with optical band-pass filters mounted in front of a 8mm C-mount lens (M118FM08, Tamron Co. Ltd., Japan). The optical filters where selected specifically to pinpoint both the amount of diffusively backscattered UV light (310-390 nm FWHM, U-360 UV, Edmund Optics Inc, US), $I_{\text {ex }}$, and the amount of AGE related autofluorescence (450-500 nm, 6 OD outside the band pass region, Edmund Optics Inc, US), $I_{e m}$. UV exposure of the tissue was performed using an 8W 365nm LED ring light (R130-365-N, Smart Vision Lights, US).

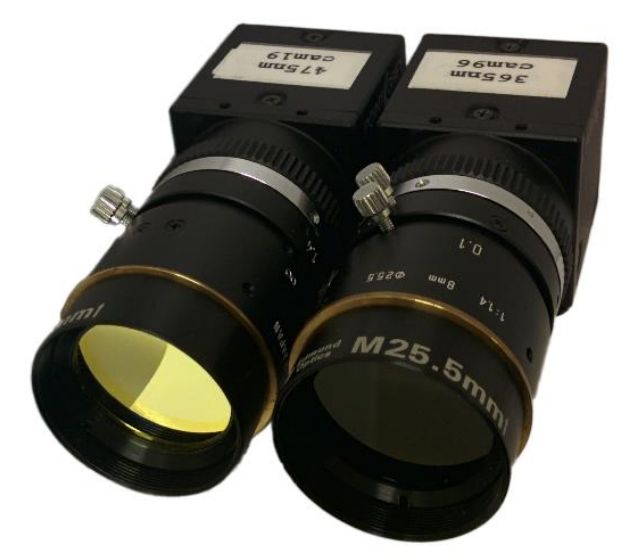

Figure 3. Two-camera MSI system with custom optical filters.

Image acquisition was carried out using a software sync scheme allowing for simultaneous acquisition of images from both cameras. For each camera a set of 10 images where recorded with the UV LED turned both off and on. The switching of the light settings was computer controlled, allowing for the acquisition off all images to be completed in less than 2 seconds. 
This rapid acquisition scheme allowed for only minor movement artifacts due to subject head or arm movement. The sets of 10 images where averaged in the post-processing to increase the SNR of the weak auto fluorescence signal.

\section{Image analysis}

An ambient light and dark current cancelation scheme was employed. This was achieved by analyzing differential images acquired while having the UV light source turned on and off, resulting in the differential excitation image

$$
\Delta I_{e x}=I_{e x, U V_{o n}}-I_{e x, U V_{o f f}}
$$

and the differential auto fluorescence image

$$
\Delta I_{e m}=I_{e m, U V_{o n}}-I_{e m, U V_{o f f}}
$$

To allow for comparing measurements performed over many days during different camera settings, calibration of the system was done using images taken from a UV exposed fluorescent object. The calibration factor $A F_{\text {ref }}$ was calculated as

$$
A F_{r e f}=\frac{\left\langle\Delta I_{e m, r e f}\right\rangle_{M_{e m}}}{\left\langle\Delta I_{e x, r e f}\right\rangle_{M_{e x}}}
$$

where $\Delta I_{\text {em,ref }}$ is the differential auto fluorescence image and $\Delta I_{\text {ex,ref }}$ is the differential excitation image, both taken from the calibration object. The area averaged intensity, indicated by $\langle\ldots\rangle_{M}$, is calculated over the mask $M_{e m}$ and $M_{e x}$, respectively. These two masks were matched in size and calculated using simple thresholding, effectively pinpointing the reference fluorescence object in the two respective images.

An adjustment of the off-axis camera position was done using a geometrical transformation algorithm. This transformation, implemented using Matlab (function fitgeotrans, MathWorks 
Inc., US), was based on local piecewise-linear regions. The local affine transformation maps for these regions were calculated using at least four manually added control points in each image pair. The control points were selected close to the region of interest in the respective image pair, i.e. the visible skin region that is later used for $A F$ quantification. Tissue regions that diverted strongly from the image plane (i.e. the region-of-interest plane), such as the nose region and the curved periphery of the face / arm, were also avoided when selecting control points.

To increase the SNR in the auto fluorescence images and to substantially lower the cost of the system, polarization filters were not employed. Instead, the images were further analyzed excluding regions of significantly higher intensity, reducing the influence from specular reflections. The non-specular regions of interest where selected in a three-step procedure using the differential excitation image, as no specular effect are per se present in the auto fluorescence image. In a last fourth step, non-representative $A F$ ratios were remove. The mask-selection procedure is described by:

1. An initial region-of-interest mask was applied to the images. For the facial images a common skin mask was used, where the nose region was excluded. The common mask was transformed to each individual using an affine transformation (translation, scaling and rotation) based on the position of the eyes. For the arm measurement, a manually selected mask of the volar side of the forearm, avoiding the curved periphery of the arm, was used.

2. High- and low-intensity pixels were avoided using image thresholding. The selection of pixels was done based on the average intensity in the initial region-of-interest mask, where pixels above $150 \%$ of the average and pixels below $50 \%$ of the average were removed from the initial mask in step 1. 
3. Border/edge pixels of the mask where avoided to further reduce the effect of specular reflections and imperfect image transformation. This was achieved by eroding the thresholded image mask in step 2 using a nine pixel wide (diameter) disk-shaped kernel.

4. Pixels displaying non-representative $A F$ ratios were removed. This was done by excluding the pixels within the eroded mask (step 3) that displayed the $20 \%$ lowest and $20 \%$ highest $A F$ ratios.

By taking the dark measurements, the calibration measurements, the image transformation and the tissue mask selection into account, an MSI AGE index was calculated as an areaaveraged normalized auto fluorescence ratio

$$
A G E_{M S I}=\frac{1}{A F_{r e f}}\left\langle\frac{\Delta I_{e m}^{\prime}}{\Delta I_{e x}}\right\rangle_{M},
$$

where $\Delta I_{e m}^{\prime}$ is the geometrically transformed auto fluorescence image. The area averaged intensity ratio, indicated by $\langle\ldots\rangle_{M}$, is calculated over the tissue mask $M$, described by the four step procedure above. Having removed the influence from specular reflections and the dependency to the excitation source, this ratio will scale to the amount of AGE in tissue. A representative example of the acquired MSI images is given in Figure 4, Figure 5 and Figure 6 below. These images show how the geometrically transformed common face mask is applied and how the mask selection procedure described above effectively removes regions with specular reflections. 


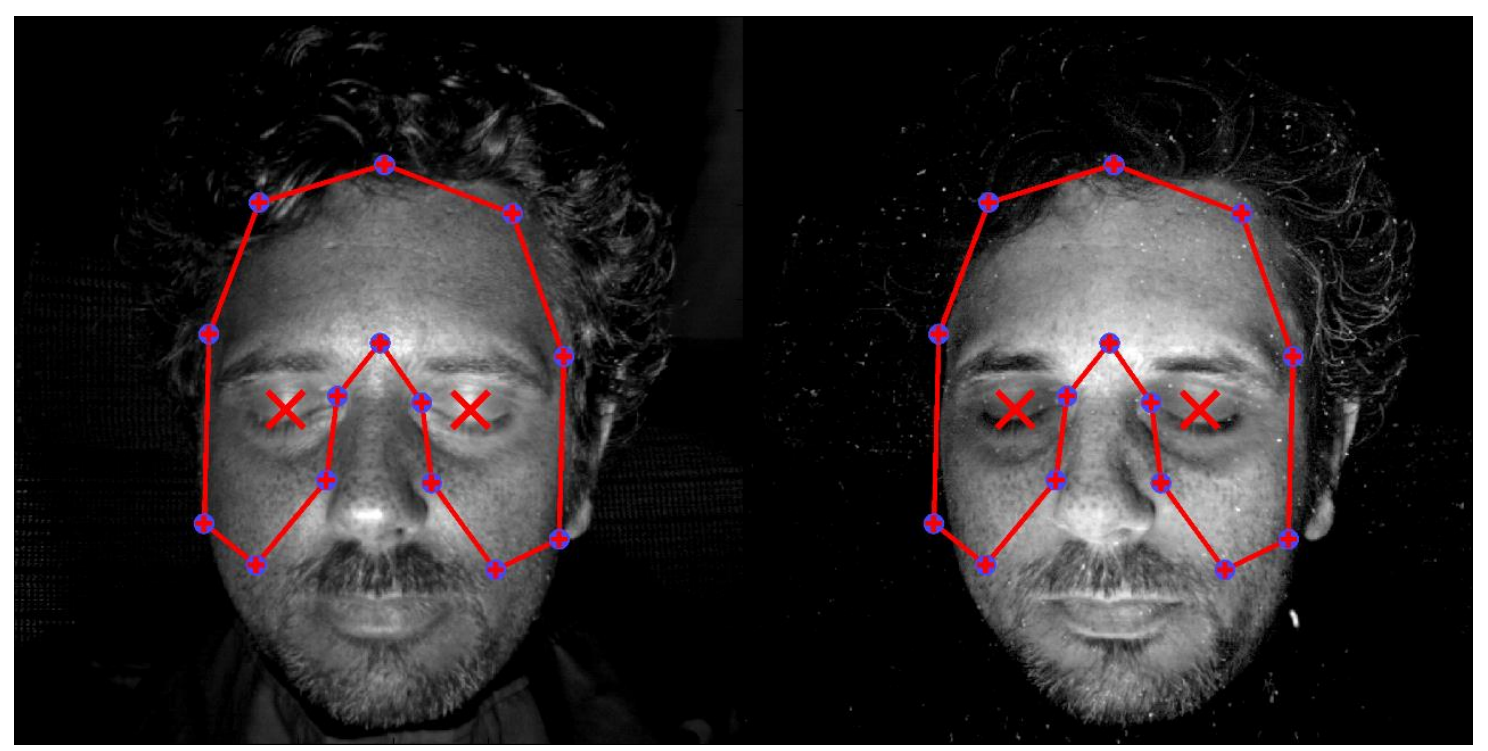

Figure 4. Example of a 310-390 nm diffuse image (left) and a 450-500 nm auto fluorescence image (right) with the geometrically transformed common face mask.
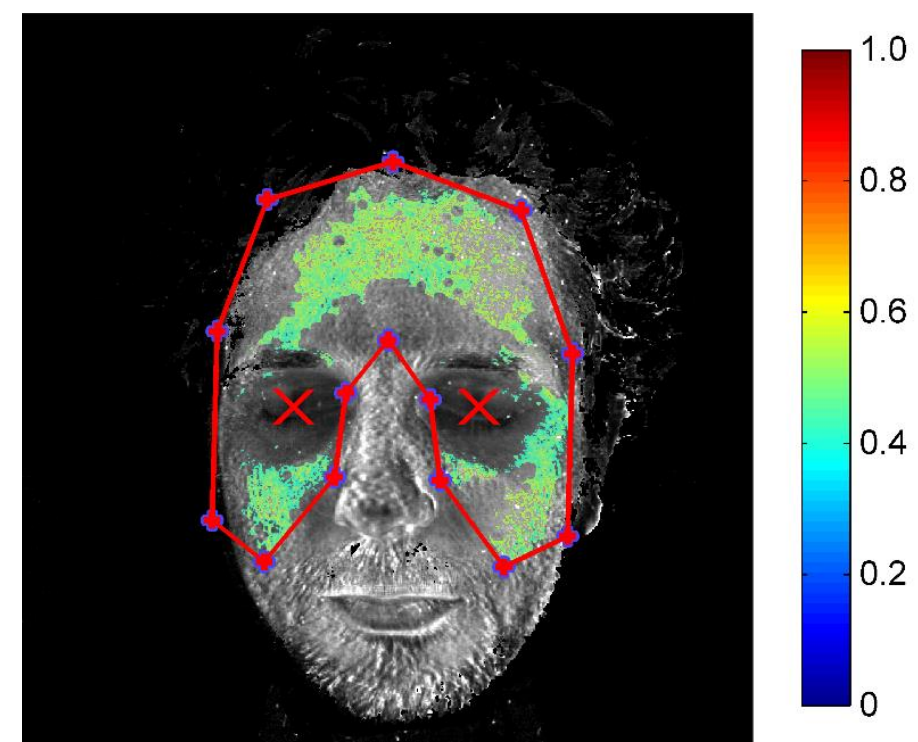

Figure 5. Example of a grayscale facial AGE index image where the overlaid colored regions indicate non-specular pixels that are automatically selected for calculating the ROI averaged AGE index. The color scale shows the AGE index of the selected pixels. 


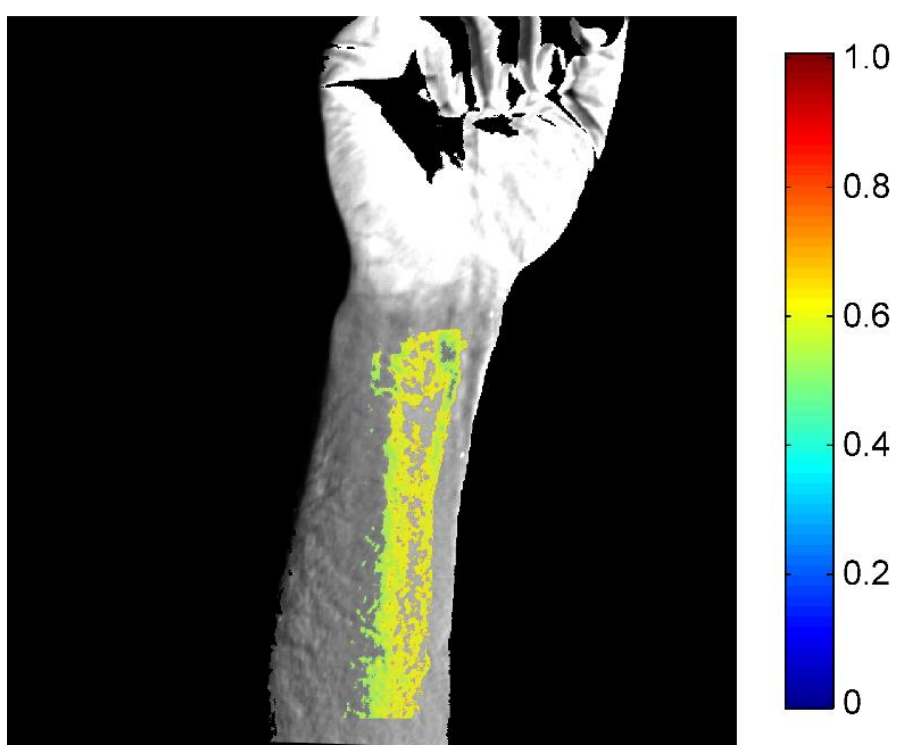

Figure 6. Example of a grayscale AGE index image of the volar side of the arm (same subject as in

Figure 5). The overlaid colored regions indicate non-specular pixels that are automatically selected for calculating the ROI averaged AGE index. The color scale shows the AGE index of the selected pixels.

\section{Material and protocol}

In total 16 subjects ( 5 female and 11 male; age: 29-61 years), having a skin color ranging from fair to deeply pigmented, were evaluated. The subjects were selected from a normal population including individuals with different cardio-metabolic diseases. The presented examination was part of a larger study aiming at evaluating the possibility of using imaging techniques for individual self-assessment and self-monitoring of cardio-metabolic risk. The clinical experimental protocol, conducted in Pisa, Italy, was approved (September 10th, 2015) by the local Ethical Committee of Pisa, Italy (Study 213/2014) and received the Ethical Clearance certification $(0086129$, November 11th, 2014) by the Italian National Commission for Research Ethics and Bioethics. Written informed consent was obtained from all subjects included in this study. 
For comparison, reference measurements using a commercially available AGE Reader (Diagnoptics Technologies B.V., Groningen, The Netherlands) were performed. The AGE reference measurements were done on a single point on the volar side of the forearm. The design of the AGE Reader did not allow for measurements of facial skin. The AGE Reader was not capable of measuring on the most deeply pigmented subject, excluding one subject in the study.

After the reference measurements the subjects were placed in a dark room where facial makeup was removed prior to any measurements taking place. The subsequent examination included skin images taken during UV exposure of the volar side of the right forearm and the facial region. For two of the subjects additional exposures (both forearm and facial) having the ambient room light turned on during image acquisition, were performed.

\section{Results}

When analyzing the correlation between the AGE Reader data and the AGE MSI data, only the 15 subjects that displayed any measures from the AGE Reader were included (one subject with the darkest skin pigmentation was excluded). The four additional recordings having the ambient light turned on were also excluded from the statistical comparisons.

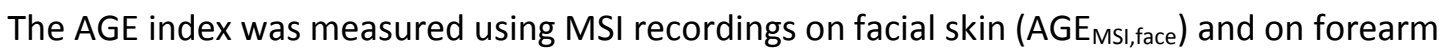
skin ( $A G E_{M S I, a r m}$ ). These indices were compared to those from the AGE Reader ( $A G E_{\text {reader }}$ ). The AGE Reader measurements were calculated as an average of two consecutive measurements. Correlation coefficients (Pearson's R) between these variables were calculated using Matlab (MathWorks Inc., US). The relationship between $A G E_{M S I, f a c e}$ and $A G E_{\text {reader }}(R=0.47, p=0.078$, $n=15)$ and the relationship between $A G E_{M S I, a r m}$ and $A G E_{\text {reader }}(R=0.68, p=0.005, n=15)$ is 


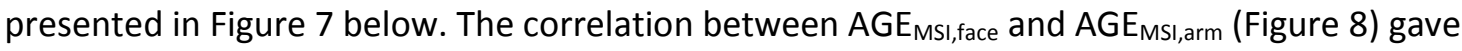
$R=0.76$ and $p=0.001(n=16)$.
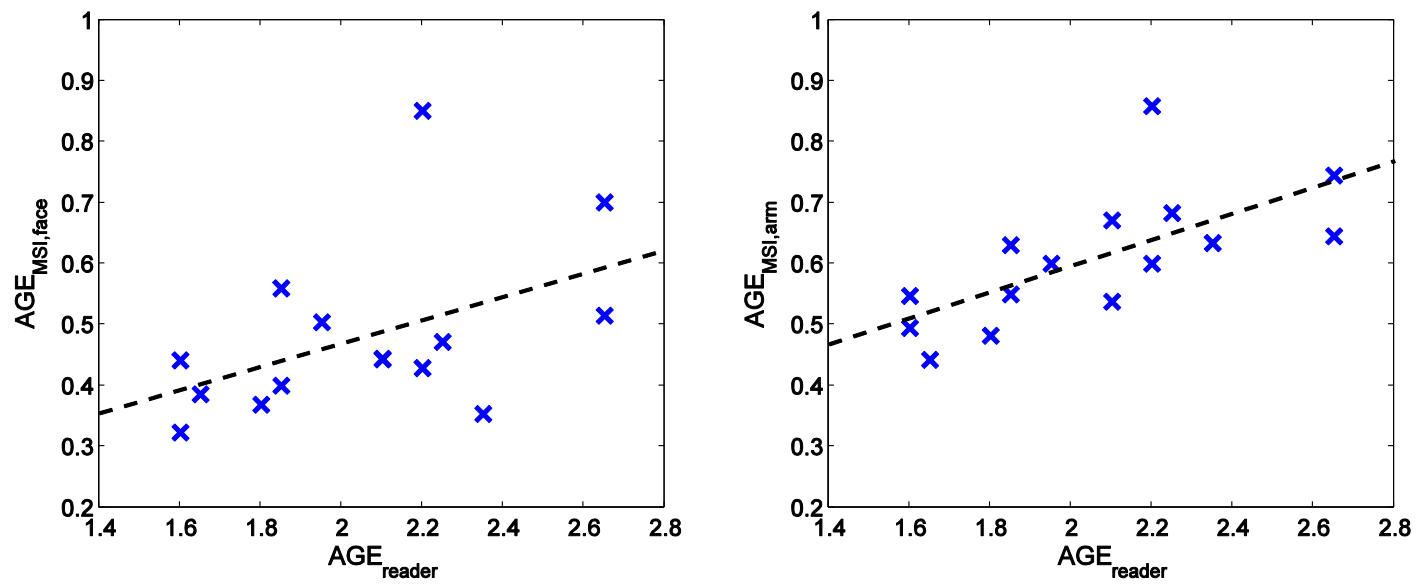

Figure 7. Left: AGE measured with MSI in facial skin (AGEMSI,face) vs AGE measured with AGE Reader (AGEreader; $R=0.47, p=0.078, n=15 ;$ Two points coincide). Right: AGE measured in forearm

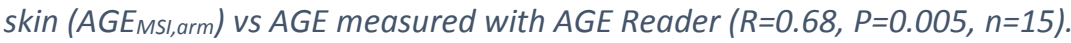

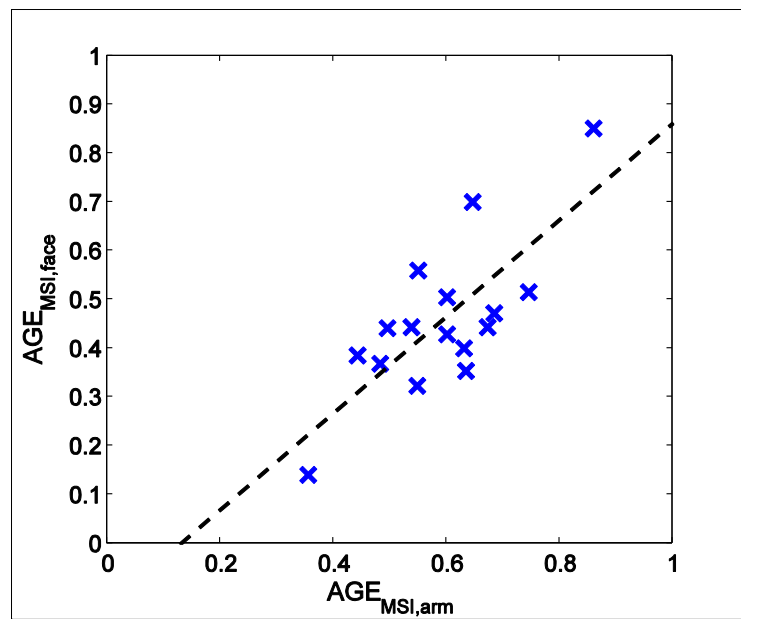

Figure 8. AGE measured with MSI in facial skin (AGEMSI,face) vs forearm skin (AGEMSI,arm; $R=0.76$, $P=0.001, n=16)$. 
For two subjects, additional AGE images were recorded with the ambient light turned on. The relative difference in AGE level in facial skin was 12.4 and $11.8 \%$, respectively. The corresponding differences between the AGE level in forearm skin were $2.5 \%$ and $5.7 \%$, respectively.

\section{Discussion}

When skin is exposed to UV light in the $350-400 \mathrm{~nm}$ range, AGE tissue compounds will start to fluoresce by re-emitting the absorbed photons as light with a longer wavelength. By detecting the auto fluorescence it is possible to track changes in the relative amount of AGE products in skin. However, the auto fluorescence is an extremely week signal when compared to the amount of elastically scattered photons. Hence it is challenging to accurately detect and quantify this signal. There is a commercialized device that quantifies AGE in forearm skin (The AGE Reader, Diagnoptics Technologies B.V., Groningen, The Netherlands) using an optical measurement in contact to the tissue. However, for an effortless and automatic assessment of larger tissue areas such as the facial skin, it would be favorable to make use of cameras and advanced algorithms.

This study is the first proof-of-principle for remote imaging of AGE in skin tissue. The results showed a significant correlation between the proposed MSI technique and the reference measurements when measuring on the volar side of the forearm $(p=0.005)$. The results also suggest that the AGE content in the facial skin area differs from that in the arm region. On average, the arm region displayed a $27 \%$ larger AGE content. However, the differences can to some extent also be explained by a higher exposure to sun light in the facial skin. This will create: 1) a larger photo-bleaching effect in facial skin; and 2) a higher photon absorption coefficient in facial skin which to a higher degree will absorb deeply penetrating photons that 
to a greater extent generates $A F$ (i.e. the penetration depth and the AF decreases with increased absorption).

The absolute levels of the reference measurements, performed with the AGE Reader, and the MSI readings cannot directly be compared as different calibrations phantoms were utilized. Still the results supports that it is possible to remotely monitor the tissue AGE auto fluorescence using an MSI camera setup. However, as indicated by the strength in the relationship between the reference measurement and the MSI readings, there is room for improvements and further studies.

One way of improving the MSI technique is to utilize polarization filters to avoid specular reflections. However, this would significantly lower the amount of detectable light resulting in a decreased SNR. This solution would also be expensive as no low-cost polarization filter exists for UV light, making the technique less attractive to a consumer market. Applying the proposed image analysis scheme for suppression of specular effects improves the correlation to the reference measurements. This shows that it is possible to decrease the specular effects without using polarization filters.

The amount of detected fluorescence depends, among other things, also on the optical properties of the skin. Tissue scattering effects and absorption of excitation or emission light by skin compounds will impact the AF measurement.[13] For example, skin AF measurements in subjects with darker skin colors typically result in lower values than in subjects with fair skin colors. In this study, the subject having the darkest skin color was the only one not displaying any reading from the AGE Reader and was subsequently excluded from the statistical analysis. In line with previous findings, this subject also displayed the lowest MSI AGE index. A method to adapt the estimation of skin AF in healthy subjects for the influence of skin color is described in Koetsier et al. [15] This approach is based on empirical optimization, using a sample population of non-diabetic subjects, of a set of correction parameters that are 
calculated from spectra in the UV-A and visible range. In an animal study, Hull et al. use a simple correction for AGE auto fluorescence, also based on empirical optimization exploiting both fluorescence and reflectance spectra. [16] Wang et al. used a similar correction in experiment on humans. [17] Similarly, to further improve the MSI AGE algorithm presented here, image data from additional spectral bands in the visible range could be added to the algorithm. This will allow for an individual correction due to variations in the skin optical properties. However, introducing additional cameras for imaging other spectral regions does not only increase the cost of the system, but also adds to the complexity of co-registration of all images (spatial alignment).

The main benefit of having a remote assessment of the AGE content is the possibility of integrating the technique into device for an effortless automatic self-assessment over time. In such a setting surrounding factors such as ambient light must be considered. By using a modulated light source and differential images we were able to significantly decrease the ambient light effect. This suggests that the proposed technique can be used in a non-dark environment.

\section{Conclusions}

AGE measured with MSI in forearm skin was significantly correlated $(p=0.005)$ with the AGE reference method (AGE Reader on forearm skin). The relationship to AGE with MSI in facial skin was slightly weaker and borderline significant $(p=0.078)$. This could indicates that the AGE content varies between forearm and facial skin areas. The recordings of AGE level with ambient light turned on showed only a minor influence of ambient light. These results show proof-of-principle for measuring AGE with the suggested MSI set-up. Longitudinal measurements are needed to determine reproducibility and also to determine the importance of the skin site used for AGE determination (face vs forearm). The main findings in this study 
indicate that it is possible to use multi-spectral imaging for non-touch assessment of skin AGE content.

\section{Acknowledgments}

This work was supported by the European Commission under the Seventh Framework

Programme for the Collaborative project under grant agreement N. 611516 (SEMEOTICONS).

The authors would like to thank Giuseppe Coppini, Sara Colantonio, Maria Aurora Morales, Paolo Marraccini and the rest of the team from CNR-ISTI and CNR-IFC for making the experimental part possible.

\section{References}

[1] A. Goldin, J.A. Beckman, A.M. Schmidt, M.A. Creager, Advanced glycation end products: sparking the development of diabetic vascular injury, Circulation, 114 (2006) 597-605.

[2] R. Singh, A. Barden, T. Mori, L. Beilin, Advanced glycation end-products: a review, Diabetologia, 44 (2001) 129-146.

[3] T. Koschinsky, C.J. He, T. Mitsuhashi, R. Bucala, C. Liu, C. Buenting, K. Heitmann, H. Vlassara, Orally absorbed reactive glycation products (glycotoxins): An environmental risk factor in diabetic nephropathy, P Natl Acad Sci USA, 94 (1997) 6474-6479.

[4] T. Goldberg, W.J. Cai, M. Peppa, V. Dardaine, B.S. Baliga, J. Uribarri, H. Vlassara, Advanced glycoxidation end products in commonly consumed foods, J Am Diet Assoc, 104 (2004) $1287-1291$.

[5] A.M. Schmidt, S.D. Yan, J.L. Wautier, D. Stern, Activation of receptor for advanced glycation end products - A mechanism for chronic vascular dysfunction in diabetic vasculopathy and atherosclerosis, Circulation Research, 84 (1999) 489-497. 
[6] A.M. Schmidt, O. Hori, J. Brett, S.D. Yan, J.L. Wautier, D. Stern, Cellular Receptors for Advanced Glycation End-Products - Implications for Induction of Oxidant Stress and Cellular Dysfunction in the Pathogenesis of Vascular-Lesions, Arterioscler Thromb, 14 (1994) 1521-1528.

[7] K. Posch, S. Simecek, T.C. Wascher, G. Jurgens, S. Baumgartner-Parzer, G.M. Kostner, W.F. Graier, Glycated low-density lipoprotein attenuates shear stress-induced nitric oxide synthesis by inhibition of shear stress-activated L-arginine uptake in endothelial cells, Diabetes, 48 (1999) 1331-1337.

[8] S.D. Yan, A.M. Schmidt, G.M. Anderson, J.H. Zhang, J. Brett, Y.S. Zou, D. Pinsky, D. Stern, Enhanced Cellular Oxidant Stress by the Interaction of Advanced Glycation End-Products with Their Receptors Binding-Proteins, Journal of Biological Chemistry, 269 (1994) 98899897.

[9] V.M. Monnier, R.R. Kohn, A. Cerami, Accelerated Age-Related Browning of Human Collagen in Diabetes-Mellitus, P Natl Acad Sci-Biol, 81 (1984) 583-587.

[10] M. Koetsier, H.L. Lutgers, A.J. Smit, T.P. Links, R. de Vries, R.O.B. Gans, G. Rakhorst, R. Graaff, Skin autofluorescence for the risk assessment of chronic complications in diabetes: a broad excitation range is sufficient, Optics Express, 17 (2009) 509-519.

[11] R. Meerwaldt, T. Links, R. Graaff, S.R. Thorpe, J.W. Baynes, J. Hartog, R. Gans, A. Smit, Simple noninvasive measurement of skin autofluorescence, Ann Ny Acad Sci, 1043 (2005) 290-298.

[12] Y. Andreu-Cabedo, P. Castellano, S. Colantonio, G. Coppini, R. Favilla, D. Germanese, G. Giannakakis, D. Giorgi, M. Larsson, P. Marraccini, M. Martinelli, B. Matuszewski, M. Milanic, M. Pascali, M. Pediaditis, G. Raccichini, L. Randeberg, O. Salvetti, T. Stromberg, Mirror mirror on the wall...; An intelligent multisensory mirror for well-being selfassessment, Multimedia and Expo (ICME), 2015 IEEE International Conference on, 2015, pp. 1-6. 
[13] R. Graaff, R. Meerwaldt, H.L. Lutgers, R. Baptist, E.D. de Jong, J.R. Zijp, T.P. Links, A.J. Smit, G. Rakhorst, Instrumentation for the measurement of autofluorescence in the human skin, P Soc Photo-Opt Ins, 5692 (2005) 111-118.

[14] A. McIntosh, B. Atshaves, H. Huang, A. Gallegos, A. Kier, F. Schroeder, Fluorescence Techniques Using Dehydroergosterol to Study Cholesterol Trafficking, Lipids, 43 (2008) $1185-1208$.

[15] M. Koetsier, E. Nur, C.M. Han, H.L. Lutgers, T.P. Links, A.J. Smit, G. Rakhorst, R. Graaff, Skin color independent assessment of aging using skin autofluorescence, Optics Express, 18 (2010) 14416-14429.

[16] E.L. Hull, M.N. Ediger, A.H.T. Unione, E.K. Deemer, M.L. Stroman, J.W. Baynes, Noninvasive, optical detection of diabetes: model studies with porcine skin, Optics Express, 12 (2004) 4496-4510.

[17] Y.K. Wang, L. Zhu, L. Zhang, G. Zhang, Y. Liu, A. Wang, A portable system for noninvasive assessment of advanced glycation end-products using skin fluorescence and reflectance spectrum, J Appl Spectrosc+, 79 (2012) 431-436. 